
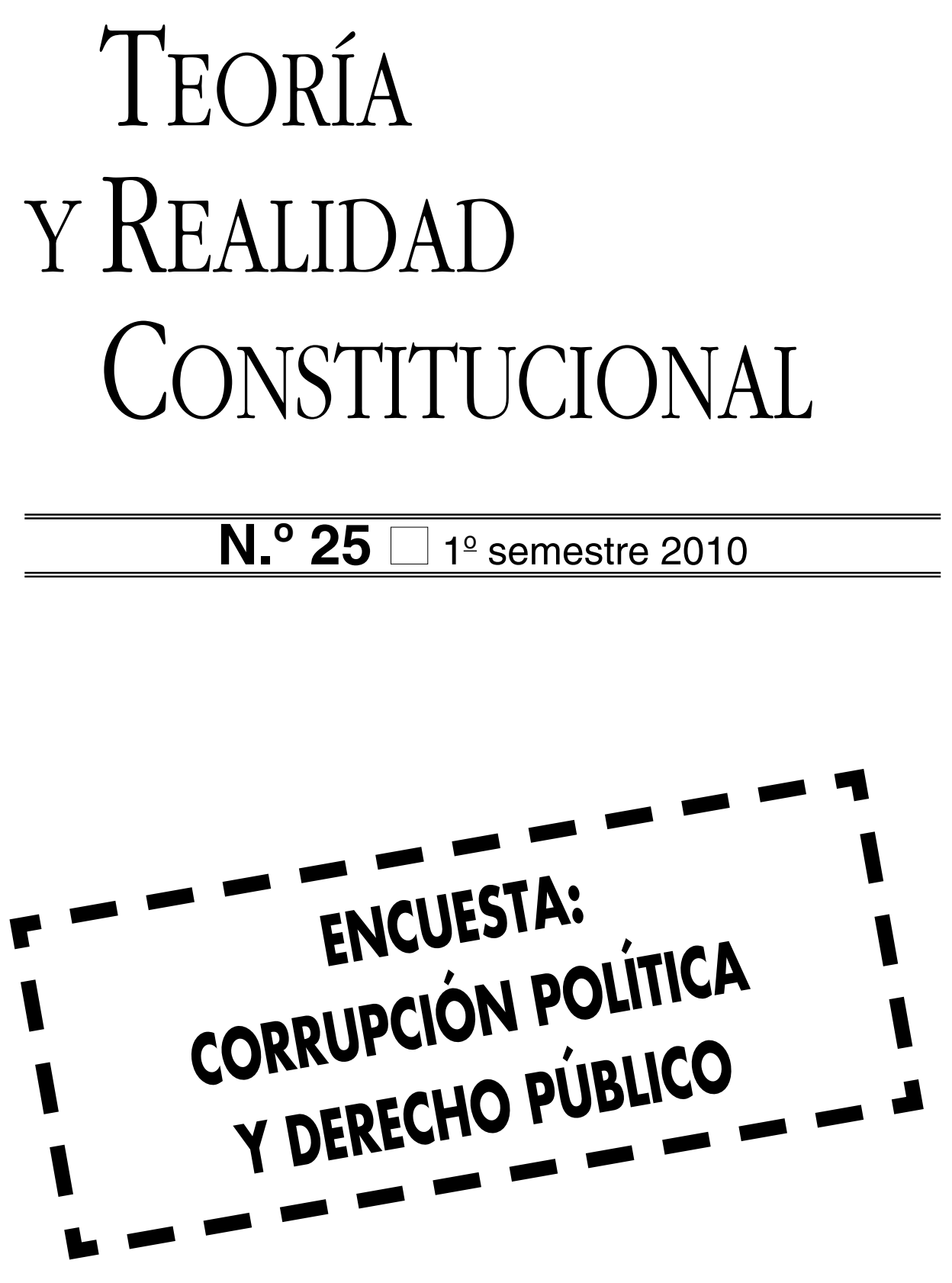


\title{
¿FORTALECIMIENTO LEGÍTIMO DEL BUNDESTAG O LECHO CONSTITUCIONAL DE PROCRUSTES? ACERCA DE LA SENTENCIA DEL TRIBUNAL CONSTITUCIONAL FEDERAL SOBRE EL TRATADO DE LISBOA*
}

\author{
CLAUS DIETER CLASSEN \\ Catedrático de Derecho Público \\ Universidad de Greifswald**
}

\begin{abstract}
SUMARIO
I. Consideraciones previas.

II. Sobre la legitimidad democrática de la UE.

III. Sobre la responsabilidad de los Parlamentos nacionales en la integración.

IV. El Estado constitucional democrático en la Unión.

V. Valoración global.
\end{abstract}

\section{CONSIDERACIONES PREVIAS}

\section{Aspectos EXTERNOS}

Después de haberse celebrado la vista oral los días 10 y 11 de febrero, la sentencia del Tribunal Constitucional Federal (en adelante, TCFA) se dictó el 30 de junio de 2009, poco antes de la pausa estival, superando de esta

* La traducción ha sido realizada por M.A. MARTín VIDA, Doctora en Derecho, investigadora en el Instituto de Derecho Público de la Goethe-Universität Frankfurt am Main (Alemania).

** El autor da clases de, entre otros, Derecho Público y Derecho de la Unión Europea en la Universidad de Greifswald (Alemania). Este artículo se publicó originalmente en alemán en el número 18 de la revista Juristenzeitung (septiembre de 2009), págs. 881-889. 
manera en más del cincuenta por ciento el plazo básico máximo de tres meses entre vista oral y sentencia previsto normativamente. Dado que a finales de septiembre se celebran elecciones generales al Bundestag y a principios de octubre el referéndum en Irlanda, los retoques a la Ley de Acompañamiento al Tratado exigidos por el Tribunal han de llevarse a cabo en sesiones extraordinarias en plena campaña electoral (si es que se quiere ratificar el Tratado antes del referéndum irlandés). Esto a su vez sería conveniente si no se quiere poner en peligro el resultado positivo del mismo, un resultado que, en vista de la decisión favorable al Tratado de Lisboa de los órganos constitucionales alemanes políticamente legitimados y de su calificación como constitucional por parte del TCFA, parece deseable desde la perspectiva alemana. Uno se habría alegrado, pues, si la (supuesta) simpatía del Tribunal hacia el Parlamento se hubiese reflejado también en su calendario de trabajo.

\section{SOBRE LA ADMISIBILIDAD}

Era previsible que un recurso de amparo iba a ser calificado de admisible, a la vista de la línea seguida por el TCFA en su sentencia "Maastricht" ${ }^{\text {. Sor- }}$ prende en cualquier caso que, dado que esto se logró mediante una revaluación del derecho de sufragio en el sentido de un derecho a un Parlamento poderoso $^{2}$, el conflicto entre órganos constitucionales paralelo fuese calificado en lo esencial de inadmisible con el argumento de que este derecho no afecta a ningún derecho del Bundestag y, en esa medida, no existe ningún interés específico de su estatus necesitado de tutela jurídica ${ }^{3}$. Esto demuestra una vez más la problemática de esta revaluación del derecho de sufragio que penetra ampliamente en la esfera del Derecho de la organización del poder público ${ }^{4}$. Sin fundamentarlo y de una manera que no resulta en absoluto convincente desde el punto de vista objetivo, el Tribunal da un paso más que en 1993 al asumir que a través del principio democrático se puedan examinar también los demás principios mencionados en el artículo 23.1.3 en conexión con el artículo 79.3 de la Ley Fundamental de Bonn (en adelante, GG), sobre todo el principio del Estado social'5.

1 Sentencia del Tribunal Constitucional Federal (en adelante, STCFA) de 30 de junio de 2009 = Juristenzeitung (JZ) 2009, 890 (en ese número en una versión acortada; una versión en inglés de la sentencia puede verse en www.bverfg.de/entscheidungen/es20090630_2bve000208en.html), marginales 168 ss., en referencia a la BVerfGE (sentencia del Tribunal Constitucional Federal) 89, 155 (171 y siguiente) = JZ 1993, 1100 (1101 y siguiente); al respecto GÖTZ, JZ 1993, 1081.

2 Véase al respecto la STCFA de 30 de junio de 2009 (nota a pie 1), marginal 240.

3 STCFA de 30 de junio de 2009 (nota 1), marginal 206 y marginal 199.

4 Sobre esta crítica véase, por ejemplo, TOMUSCHAT, Europäische Grundrechte Zeitschrift (EuGRZ) 2003, 489 (489 ss.).

5 STCFA de 30 de junio de 2009 (nota 1), marginal 182. 


\section{CONSIDERACIONES PREVIAS RESPECTO AL CONTENIDO}

Antes de abordar las reflexiones centrales del Tribunal en relación a la democracia y a la estatalidad constitucional hay que destacar algunas otras declaraciones del TCFA. De manera detallada se argumenta la necesidad de un fundamento de Derecho internacional convencional para la Unión ${ }^{6}$. Sin embargo no se discute que éste existe también ahora. De hecho, todo el Tratado debía volver a eliminar la cercanía de la UE a la condición de Estado que establecía el Tratado constitucional ${ }^{7}$; ni siquiera desde una perspectiva fáctica considera el TCFA a la Unión como un Estado federal, tampoco después de Lisboa. Por eso resultan superfluas las consideraciones acerca de las condiciones constitucionales básicas para la fundación de un Estado federal (que en la actualidad tampoco en otros Estados miembros de la UE podría ni remotamente lograr la mayoría necesaria), en concreto la referencia al artículo 146 GG y a las condiciones-marco de su aplicación (es necesario un referéndum; el sentido del artículo 79.3 GG se deja abierto $^{8}$ ). Al menos se reconoce al mismo tiempo funcionalmente carácter constitucional a los Tratados comunitarios $^{9}$. Si bien eso ya lo había hecho el TCFA en los años sesenta ${ }^{10}$, no deja de ser, sin embargo, una destacable evolución desde la perspectiva de la sentencia "Maastricht", en la que este concepto no se empleó ${ }^{11}$.

La idea clave de la "simpatía hacia Europa" de la Ley Fundamental es nue$\mathrm{va}^{12}$. No se concreta más detalladamente en el presente caso, pero al menos abre una puerta. A la vista de su sentencia "Görgülü»"13, el TCFA debería denunciar en el futuro graves fallos en la aplicación del Derecho de la UE por parte de instancias alemanas, también más allá del ámbito de aplicación del artículo 101 GG en conexión con el artículo 234.3 TCE (artículo 267.3 del Tratado sobre el Funcionamiento de la UE, en adelante TFUE), y seguir de este modo en esa medida al Conseil constitutionnel francés, que ha derivado de una disposición de la Constitución francesa comparable al artículo 23 GG una facultad análoga ${ }^{14}$.

6 STCFA de 30 de junio de 2009 (nota 1), marginales 228 ss.

7 STCFA de 30 de junio de 2009 (nota 1), marginal 277.

8 STCFA de 30 de junio de 2009 (nota 1), marginales 217, 228, 231 y siguiente.

9 STCFA de 30 de junio de 2009 (nota 1), marginal 231.

10 BVerfGE 22, 293 (296).

11 Una de las causas de ello fue muy probablemente la decidida posición contraria del entonces Magistrado y Ponente P. KIRCHHOF (por ejemplo en Festschrift Oppermann, 2001, pág. 201 [212 y siguiente]).

12 STCFA de 30 de junio de 2009 (nota 1), marginal 225.

13 BVerfGE 111, 307 (316 ss.).

14 Conseil constitutionnel 2004-496 DC, traducción al alemán en JZ 2004, 969 con comentario de CLASSEN (en relación al artículo 88-1 de la Constitución francesa). 


\section{SOBRE LA LEGITIMIDAD DEMOCRÁTICA DE LA UE}

\section{Fundamentos GENERALES}

Cuando el TCFA aborda las exigencias de la legitimidad democrática de la UE a los efectos del artículo 23.1.1 GG no sólo las sustenta, con razón, sobre el principio nacional democrático en el sentido de los artículos 20 y $28 \mathrm{GG}^{15}$, sino que tiene también en cuenta las tradiciones de otros Estados miembros $^{16}$, así como las particularidades de la estructura de una «asociación de Estados" (Staatenverbund) ${ }^{17}$. Al final se califica a la Unión de democrática. Para llegar a esta conclusión no es necesaria, sin embargo, la detallada descripción del déficit democrático existente desde el punto de vista estatal ${ }^{18}$. Habría bastado la alusión a que las estructuras de legitimación han de estar en consonancia con la magnitud de la integración y que, por tanto, también de hecho, el alcance de las competencias de la UE no necesita justificar ninguna similitud estatal ${ }^{19}$.

\section{Problemas Del PRINCIPIO MAYORITARio}

Antes de abordar los problemas específicos de la integración europea, el TCFA hace algunas reflexiones generales acerca del principio democrático. De acuerdo con ellas hay que "poder articular, en la elección de la asamblea representativa del pueblo o en la elección de los altos cargos del Gobierno ... una voluntad mayoritaria, personal u objetivamente generalizada, e inferir de la elección decisiones acerca de la orientación política”.

En el sistema alemán se garantiza con ello que las decisiones mayoritarias en el Parlamento representen a su vez decisiones mayoritarias del pueblo ${ }^{20}$. Por el contrario, en la UE falta un sistema de organización gubernamental en el que una voluntad europea mayoritaria sustente de tal modo la formación de un Gobierno que se remonte a una decisión electoral libre e igual y que permita hacer surgir una auténtica rivalidad, transparente para el ciudadano, entre Gobierno y oposición ${ }^{21}$. "En el caso de decisiones ajustadas entre orientaciones políticas en el Parlamento europeo no hay ninguna garantía de que la mayoría de los votos emitidos representa también a una mayoría de los ciudadanos de la Unión "22.

15 STCFA de 30 de junio de 2009 (nota 1), marginales 219, 227.

$16 \mathrm{Al}$ respecto STCFA de 30 de junio de 2009 (nota 1), marginales 214, 269.

17 STCFA de 30 de junio de 2009 (nota 1), marginales 266 y siguiente, 271 y siguiente.

$18 \mathrm{Al}$ respecto STCFA de 30 de junio de 2009 (nota 1), marginales 289-297.

$19 \mathrm{Al}$ respecto STCFA de 30 de junio de 2009 (nota 1), marginales 262, 264.

20 STCFA de 30 de junio de 2009 (nota 1), marginales 213 y siguiente.

21 STCFA de 30 de junio de 2009 (nota 1), marginal 280.

22 STCFA de 30 de junio de 2009 (nota 1), marginal 281. 
A esto se puede oponer, sin embargo, que, debido a la cláusula del cinco por ciento (el porcentaje que un partido tiene que alcanzar para lograr representación en el Parlamento) y a los escaños adicionales extraordinarios (Überhangmandate), tampoco en Alemania el Parlamento representa siempre la voluntad mayoritaria. Además, las campañas electorales no vienen marcadas por un modelo bipolar como se conoce, por ejemplo, en Gran Bretaña y Francia. Desde 1949 los cambios de Gobierno a nivel federal han tenido lugar sólo en 1998 directamente mediante las elecciones; en el resto de los casos ha ocurrido por cambios de coalición (no anunciados antes de la elección correspondiente). Por el contrario, desde 2004 la proporción de fuerzas políticas en el Parlamento Europeo influye de manera considerable en la formación de la Comisión. En ambos sistemas, por tanto, las elecciones resultan solamente determinantes en la composición del Parlamento, mientras que la obtención de la mayoría se basa de manera decisiva en una propia y particular formación de la voluntad, independiente de las elecciones, dentro de este órgano. Precisamente aquí se sitúa el rasgo central característico de una democracia representativa. La contenida valoración de la legitimidad de la integración europea que lleva a cabo el TCFA se basa, pues, en ideas que tampoco en Alemania se materizalizan de esa manera. Si hubiese un sistema electoral mayoritario como el que hay en el Estado miembro de la UE Reino Unido las distorsiones serían todavía mayores.

\section{Acerca de la representación por el Parlamento Europeo}

También por lo demás se le hace difícil al TCFA la cuestión del Parlamento Europeo. La fórmula de su papel (sólo) complementario, acuñada en la sentencia "Maastricht" pero que no se utilizó en la sentencia sobre la orden de detención europea ${ }^{23}$, experimenta ahora un resurgimiento ${ }^{24}$. Por lo que respecta al sujeto de representación, si bien el TCFA observa que el artículo 10 TUE menciona a las ciudadanas y ciudadanos de la Unión como tales ${ }^{25}$, no se trata en ese caso, sin embargo, de un pueblo europeo (que no existe, eso no se discute). Detrás de ello hay más bien una concepción de la democracia que se retrotrae con más fuerza al individuo y en menor medida a una colectividad, definida ésta como se quiera ${ }^{26}$. Se podría dudar naturalmente del poder de convicción para la Ley Fundamental de Bonn de una concepción tal

23 BVerfGE 113, 273 (301).

24 STCFA de 30 de junio de 2009 (nota 1), marginal 262, en referencia a la BVerfGE 89, 155 (184).

25 STCFA de 30 de junio de 2009 (nota 1), marginales 279, 349.

$26 \mathrm{Al}$ respecto, por ejemplo, V. BOGDANDY, Gubernative Rechtssetzung, 2000, pág. 31; KOTZUR, en BAUER/HUBER/SOMMERMANN (eds.): Demokratie in Europa, 2005, pág. 351 (360 ss., 382 ss.); especialmente acerca de la UE PERNICE, Veröffentlichungen der Vereinigung der Deutschen Staatsrechtslehrer (VVDStRL) 60 (2001), 148 (166 y siguiente). 
de la democracia ${ }^{27}$. En todo caso no ha sido ningún problema para otros Tribunales constitucionales concebir al Parlamento como representación de los ciudadanos de la Unión ${ }^{28}$. Frente a ello el TCFA menciona la idea a través de la referencia a la dignidad humana como componente fundamental también de la democracia ${ }^{29}$, pero la ignora en el fondo y le atribuye en su lugar al Tratado un modelo propio al concebir al Parlamento Europeo, como hasta ahora, como representación de los pueblos ${ }^{30}$. Errónea resulta, por lo demás, la asunción del TCFA de que la representación se vincula a la nacionalidad ${ }^{31}$. De hecho, los ciudadanos de la Unión pueden a partir del Tratado de Maastricht votar también en su lugar de residencia (artículo 22.2 TFUE = artículo 19.2 TCE) ${ }^{32}$. Para hacerlo correctamente, al buscar una denominación adecuada para la colectividad de los representados se tendría que haber ido más allá del simple modelo bipolar (representación de un pueblo o de varios pueblos); se podría haber hablado quizá de la representación de una "unión de los pueblos" (cfr. también el artículo 1.2 TUE en su nueva versión).

\section{Sobre la igualdad Del VOtO}

Desde hace tiempo se viene criticando la calidad de la legitimidad del Parlamento Europeo a causa de las cuotas de escaños asignadas a cada uno de los Estados y de la desigualdad, asociada a ello, de la representación de los habitantes de los Estados miembros de la UE. Por eso se aborda detalladamente la cuestión de la igualdad del voto, considerada a nivel nacional, con razón, como fundamental ${ }^{33}$. En este punto se puede constatar un resultado similar al de la cuestión fundamental de la legitimidad de la UE: es correcto el resultado según el cual las desigualdades se pueden explicar en atención a las particularidades de un Parlamento en una asociación de Estados, pero se exagera al exponer en detalle los argumentos. De manera algo contradictoria se designa el principio "one man, one vote" como expresión de la "igualdad de oportunidades de éxito", que aquí no se respeta por culpa de las cuotas de escaños, mientras que más adelante, al hablar de las "oportunidades de victoria", se consideran expresamente aceptables, desde una perspectiva común europea, ciertas excepciones al principio de igualdad ${ }^{34}$. A ese respecto insiste el

27 CLASSEN, Demokratische Legimitation im offenen Rechtsstaat, 2009, págs. 28 ss.

28 Conseil constitutionnel, 2003-468 DC de 3 de abril de 2003, cons. 37; Tribunal constitucional polaco, Europarecht (EuR) 2006, 236 (237).

29 STCFA de 30 de junio de 2009 (nota 1), marginal 211.

30 STCFA de 30 de junio de 2009 (nota 1), marginales 229, 279, 284, 349; además, marginal 286, con la referencia a las situaciones de poder en cada uno de los Estados miembros.

31 STCFA de 30 de junio de 2009 (nota 1), marginal 287.

$32 \mathrm{Al}$ respecto por ejemplo CLASSEN, Archiv des öffentlichen Rechts (AöR) 119 (1994), 238 (248).

33 STCFA de 30 de junio de 2009 (nota 1), marginales 281 ss.; sobre su significado a nivel nacional además marginal 215.

34 STCFA de 30 de junio de 2009 (nota 1), marginal 279 por un lado, marginal 283 por otro. 
Tribunal en que a nivel nacional, por lo general, sólo se aceptarían grandes desequilibrios en la representación para el caso de una segunda Cámara de designación federal, pero no para una (primera) Cámara que haya de representar al conjunto de los electores ${ }^{35}$.

Frente a ello, en la cuna de la democracia, el Reino Unido, existe todavía, tras una reforma llevada a cabo justo antes de las últimas elecciones a la Cámara Baja, una cuota de escaños que apenas puede considerarse "insignificante ${ }^{36}$ : Inglaterra, con unos cincuenta millones de habitantes, está representada por quinientos veintinueve diputados, mientras que Gales, con dos millones novecientos mil habitantes, lo está por cuarenta. También la Constitución española se aparta para las elecciones a la Cámara Baja (artículo 68.2) de la igualdad del voto. El Derecho alemán especial para las minorías nacionales (no se aplica la cláusula del cinco por ciento) influye regularmente en la conformación de la mayoría en el Parlamento regional de Schleswig-Holstein. Con carácter complementario se menciona la rígida legislación francesa sobre paridad electoral de género. Si bien todas estas diferencias no son comparables a las que existen entre cada una de las cuotas por país en relación al peso de la población respectiva, estos ejemplos muestran, no obstante, que la Unión recurre en este aspecto a un instrumento sin duda conocido a nivel nacional.

\section{SOBRE LA RESPONSABILIDAD DE LOS PARLAMENTOS NACIONALES EN LA INTEGRACIÓN}

\section{FundAMENTOS}

El TCFA destaca con razón que, ni siquiera tomados conjuntamente, podrían por sí solos el Parlamento Europeo, por falta de un pueblo europeo unitario y por las desigualdades que de ahí se derivan en la conformación del voto, y el Consejo Europeo o el Consejo de Ministros, en tanto que órganos integrados sólo por representantes de los Ejecutivos, aportar una suficiente legitimidad democrática a la Unión. Por esa razón les corresponde a los Parlamentos nacionales un papel importante ${ }^{37}$ y reforzarlo constituye ciertamente una preocupación importante para el Tratado de Lisboa. Ahora bien: desde la perspectiva de la Constitución francesa las facultades reconocidas actualmente a los Parlamentos nacionales van en parte incluso más allá de lo que (hasta ahora) permitía el Derecho constitucional allí vigente $^{38}$.

35 STCFA de 30 de junio de 2009 (nota 1), marginal 286.

36 STCFA de 30 de junio de 2009 (nota 1), marginal 281.

37 STCFA de 30 de junio de 2009 (nota 1), marginal 297.

38 Conseil constitutionnel, 2007-560 DC, de 20 de diciembre de 2007. Entretanto la Constitución ha sido reformada en ese sentido. 
La "responsabilidad de los Parlamentos nacionales en la integración" postulada por el TCFA se refiere, no obstante, sólo a la transferencia de derechos de soberanía. En el caso de una decisión en este sentido habrá que examinar si la legitimidad democrática de la UE posee la calidad que exige el paso previsto en el proceso de integración ${ }^{39}$. Además, las competencias transferidas a la Unión han de estar suficientemente determinadas; en el caso de cláusulas abiertas el Parlamento nacional tiene, pues, una especial responsabilidad (2.). Además, éste asume una gran importancia en relación a las cláusulas evolutivas del Derecho de la Unión, las "cláusulas-pasarela" y las llamadas "de freno de emergencia" (Notbremseklauseln) (3.). Por el contrario, el papel del Bundestag y del Bundesrat en la producción normativa cotidiana de la Unión, esencialmente más importante para la legitimidad de la UE, no se menciona apenas (4.).

\section{Cuestiones Generales aCERCA DE la transferencia De Derechos DE SOBERANÍA}

a) Sobre la delimitación de competencias

En el punto de intersección entre la cuestión relativa a la legitimidad democrática de la UE y la relativa a la conservación de la democracia nacional se sitúa la cuestión de la delimitación suficientemente clara de las competencias. La UE es considerada (con razón) democrática sólo debido a que se fundamenta en el principio de la atribución restringida de competencias ${ }^{40}$; también la preservación de estructuras democráticas a nivel nacional presupone este principio. Destacables en este punto resultan las referencias del TCFA a la dinámica propia, anclada en esa medida también en la Ley Fundamental y por ella legitimada, inherente a una comunidad integrativa como la UE. Hay que destacar en este contexto cómo se desdramatizan los dos conceptos que aparecen sobre todo mencionados a este respecto en el debate, el de "poderes implícitos" (implied powers) y el de "efecto útil» (effet utile), de los cuales el primero se retrotrae a su origen estadounidense y a su asunción por el Tribunal Internacional de Justicia y el otro asimismo a sus orígenes en el Derecho internacional ${ }^{41}$.

En el contexto del aseguramiento de los límites competenciales menciona el TCFA también la posibilidad de recurso que se reconoce a los Parlamentos nacionales en el artículo 8 del Protocolo sobre la aplicación del principio de subsidiariedad. Esta posibilidad se limita expresamente, según el tenor literal del precepto, al principio de subsidiariedad. A pesar de ello indica el TCFA que una extensión del control judicial a la cuestión (previa) de la existencia

39 STCFA de 30 de junio de 2009 (nota 1), marginales 319, 413.

40 STCFA de 30 de junio de 2009 (nota 1), marginales 234 ss., 298 ss.

41 STCFA de 30 de junio de 2009 (nota 1), marginal 237. 
de una competencia sería útil para la efectividad de este procedimiento de control $^{42}$, lo cual es cierto pero a la vez implica una expansión de la competencia del TJCE no amparada por el tenor literal de la norma.

b) El principio de la atribución restringida de competencias

y las normas competenciales abiertas

El TCFA se ocupa en especial de determinadas normas, probablemente demasiado abiertas, del Tratado de la Unión. En el caso de la cláusula de flexibilidad del nuevo artículo 352 TFUE (antiguo artículo 235 TCEE y actual artículo 308 TCE), la valoración en principio positiva del Tribunal hacia el mantenimiento del principio de la atribución restringida de competencias se topa con un límite ${ }^{43}$. En este punto el análisis de esta norma es absolutamente acertado: dado que la norma ya no se vincula a la noción del "mercado común", ciertamente abierta pero en esencia referida al ámbito de la economía, sino, a partir de ahora, al "ámbito de las políticas definidas en los Tratados", encierra esta norma un salto cualitativo en la evolución jurídica europea ${ }^{44}$. La consecuencia (es decir, que ya no está amparada por el artículo 23.1.2 GG) es comprensible, si bien no tiene parangón en Derecho comparado; la salida de no declarar inconstitucional el Tratado sino considerar el recurso a esta competencia como una nueva transferencia de derechos de soberanía y, de este modo, someterla a una reserva de $l e y^{45}$ resulta convincente. A la vista de la unanimidad que requiere la norma no hay tampoco que temer en este sentido ningún efecto disfuncional.

Respecto a las consecuencias prácticas de la sentencia, no resulta obligatorio al respecto que el proyecto que se vaya a fundamentar en el artículo 352 TFUE haya de haberse negociado ya de forma definitiva. Dado que en este punto no se podrían plantear mayores exigencias que las que, por lo demás, ya existen en relación al grado de determinación en el caso de la transferencia de derechos de soberanía, resulta suficiente con que la ley describa a grandes rasgos el ámbito de regulación, de modo que al Gobierno Federal le quede también un cierto margen de negociación. Ahora bien, desde el punto de vista de la política jurídica no es posible a estos efectos hallar una justificación objetiva para la necesidad en todo caso, sin excepción, del voto favorable del Bundesrat según el artículo 23.1.2 GG. Mientras que en el caso de los Tratados de integración es absolutamente aceptable la exigencia de este voto favorable (que se aparta de lo establecido en el artículo 24.1 GG), a la vista de su significación constitucional y de los múltiples efectos que cabe esperar sobre las competencias del Bundesrat o de los Länder, eso mismo no

42 STCFA de 30 de junio de 2009 (nota 1), marginal 305.

43 STCFA de 30 de junio de 2009 (nota 1), marginales 325 ss.

44 OPPERMANN/CLASSEN/NETTESHEIM, Europarecht, 4. ed., 2009, marginal 6/44.

45 STCFA de 30 de junio de 2009 (nota 1), marginal 417. 
ocurre aquí. Lo mismo se puede afirmar por lo demás también de la reserva de ley requerida para ampliar la competencia en materia de Derecho penal según el artículo 83.1.3 TFUE ${ }^{46}$.

Resulta llamativo finalmente que la competencia en materia de mercado interior del artículo 114 TFUE (hasta ahora artículo 95 TCE) no se mencione en la sentencia, a pesar de que esta norma fue aplicada por el TJCE con una generosidad discutible precisamente en la sentencia de 10 de febrero de 2009, relativa a la Directiva sobre conservación de datos ${ }^{47}$, en un caso extremadamente sensible desde el punto de vista constitucional tanto en cuanto al contenido $^{48}$ como a la naturaleza de la competencia (cercana al Derecho procesal penal).

\section{Cláusulas evolutivas, "Cláusulas pasarela" y Cláusulas de "Freno DE EMERGENCIA"}

a) ¿Qué es una transferencia de derechos de soberanía?

Según el artículo 48.6 TUE en su nueva redacción podrá modificarse la Tercera Parte del TFUE, relativo a las políticas y acciones internas de la Unión, mediante una decisión del Consejo Europeo que precisará de la aprobación por parte de los Estados Miembros de conformidad con sus respectivas normas constitucionales. En cualquier caso no podrán ampliarse las competencias de la UE. Existen disposiciones similares para algunos ámbitos especiales $^{49}$.

Con este trasfondo, el TCFA, sin intentar una subsunción en la norma, simplemente aludiendo a la "preservación de la responsabilidad en la integración y para conservar la estructura constitucional", defiende que el artículo 23.1.2 GG "comprende toda modificación de los fundamentos textuales del Derecho europeo originario" "50; sólo en la medida en que las "cláusulas-pasarela" especiales se limiten a ámbitos suficientemente determinados por el Tratado de Lisboa no será imprescindible una ley a los efectos del artículo 23 $\mathrm{GG}^{51}$. La primera afirmación mencionada sorprende, porque la responsabilidad del legislador en la integración podría asegurarse también a través del

46 A este respecto STFCA de 30 de junio de 2009 (nota 1), marginal 363.

47 STJCE, asunto C-301/06 - Irlanda/Parlamento y Consejo = JZ 2009, 466.

48 Véase la BVerfGE 121, 1 (16), con alusiones al procedimiento no decidido entonces todavía por el TJCE.

49 Artículo 42.2.1 TUE, sobre la defensa común; artículo 25.2 TFUE, sobre los derechos de los ciudadanos de la Unión; artículo 218.8.2.2 TFUE, sobre la adhesión de la UE al Convenio Europeo de Derechos Humanos; artículo 233.1.2 TFUE, sobre el procedimiento electoral para el Parlamento; artículo 262 TFUE, sobre la propiedad intelectual o industrial; y artículo 311.3 TFUE, sobre los recursos propios de la Unión.

50 STCFA de 30 de junio de 2009 (nota 1), marginal 243.

51 STCFA de 30 de junio de 2009 (nota 1), marginal 320. 
artículo 23.1.3 GG o del artículo 59.2 GG. En relación a la protección de la estructura constitucional, la tercera frase del artículo $23.1 \mathrm{GG}$ resulta incluso más aproximada que la segunda frase. Allí, es decir, en la tercera frase, se halla además la diferenciación expresa entre "reformas" del Tratado y "normativa análoga"; con ello se estaba haciendo referencia a las "cláusulas-pasarela" y a las cláusulas evolutivas análogas contenidas en el Tratado de Maastricht.

Que el artículo 59.2 GG pasa a segundo plano respecto al artículo 23.1.2 GG en tanto que lex specialis ${ }^{52}$ resulta convincente, pero sólo en el marco del ámbito de aplicación de esta última norma. Ésta comprende, no obstante, solamente la transferencia de derechos de soberanía. Bajo esta noción se entiende hasta ahora sólo la atribución de derechos de soberanía autónomos, en otras palabras, competencias propias. Pero precisamente eso falta en el caso del artículo 48.6 TUE que aquí se aborda, dado que ahí el aumento de competencias se excluye expresamente. El artículo 23.1.3 GG resultaría pertinente todo lo más sólo cuando se tratase materialmente de una reforma de la Ley Fundamental. Para el resto de los supuestos se abriría la vía del artículo 59.2 GG. Que a esto se opone la ausencia de un Tratado lo desmiente la jurisprudencia del TCFA en relación a las resoluciones del Consejo de la OTAN ${ }^{53}$. Pero el planteamiento del TCFA es rechazable sobre todo porque ya no permite distinguir qué habrá que entender en el futuro por "transferencia de derechos de soberanía" a los efectos del artículo 23.1.2. Según una interpretación literal de la sentencia queda englobada incluso la reducción de normas competenciales (o el paso de una mayoría cualificada a la unanimidad) y dependería, de acuerdo con eso, de la aprobación del Bundestag y del Bundesrat.

Las reformas materiales de los Tratados podrían quizá incluso subsumirse en el artículo 23.1.2 GG por las consecuencias que conllevan en relación a las competencias jurisdiccionales del TJCE a ellas vinculadas. Respecto a las normas institucionales podría pensarse (no tanto por el tenor literal, sino a lo sumo por el telos de la norma) en aplicar la reserva de ley del artículo 23.1.2 GG si se modifican los procedimientos decisorios a través de los cuales se ejercitan los derechos de soberanía transferidos o si se alteran, por ejemplo mediante Tratados de adhesión, las correspondientes condiciones marco. Por supuesto esto debería valer siempre, también por ejemplo en caso de una adhesión de Islandia a la UE, pero sobre todo igualmente para el artículo 24.1 GG. Alemania no podría, pues, transferir derechos de soberanía a organizaciones internacionales si la adhesión a las mismas es posible también sin la aprobación de todos sus miembros. La adhesión al Estatuto del Tribunal Internacional de Justicia habría sido, según esto, inconstitucional. En este punto el TCFA puede apoyarse en que, según el Conseil constitutionnel francés,

52 STCFA de 30 de junio de 2009 (nota 1), marginal 312, en referencia (pero inútil a estos efectos) a la BVerfGE 89, 155 (199).

53 BVerfGE 104, 151 (199 ss.); de otra opinión PERNICE, en: DREIER (ed.), Grundgesetz, vol. II, 2. ed., 2006, Art. 23, marginal 86. 
hay una diferencia fundamental entre que las decisiones internacionales se adopten por unanimidad o por mayoría ${ }^{54}$, pero en ese caso el parámetro de valoración es la soberanía, expresamente recogida en la Constitución de ese país (artículo 3), más concretamente las "conditions essentielles de l'exercice de la souveraineté. El texto de la Ley Fundamental, sin embargo, no contiene ninguna garantía similar. Gertrude Lübbe-Wolf, una de las Magistradas que han participado en la emisión de la sentencia, ha alegado con razón que la unanimidad, precisamente por el efecto que tiene de dificultar la toma de decisiones, puede incluso provocar limitaciones de la soberanía, por ejemplo si las sentencias del TJCE en materia de mercado interior, que de acuerdo con la naturaleza de la materia sólo pueden producir una integración negativa, han de ser suavizadas mediante medidas de acompañamiento ${ }^{55}$. También resultan difíciles de modificar los actos una vez emitidos.

En la práctica, el recurso al artículo 23.1.2 GG en lugar de al artículo 59.2 GG tienen como consecuencia, además, que el Bundestag resulta debilitado porque sólo de acuerdo con la norma citada en primer lugar puede decidir sin el voto favorable del Bundesrat. El TCFA ha perdido aquí evidentemente de vista que el procedimiento versa originariamente sobre los derechos del Bundestag ${ }^{56}$. La posición del TCFA implica, en definitiva, que la condición "transferencia de derechos de soberanía" sea sustituida por el elemento contenido en la tercera frase "reformas de los Tratados constitutivos y normativa análoga”. Esto tiene poco que ver con una interpretación constitucional metodológicamente correcta.

b) Acerca de las "cláusulas-pasarela"

Las llamadas "cláusulas-pasarela" permiten modificaciones en el ámbito de los procedimientos de toma de decisiones. Según el artículo 48.7 TUE, el Consejo Europeo puede acordar pasar de la unanimidad a la toma de decisiones por mayoría cualificada, o de un procedimiento legislativo especial al procedimiento legislativo ordinario. Esta decisión entrará en vigor cuando en un plazo de seis meses ningún Parlamento nacional se haya opuesto a ella. Lo mismo vale, de acuerdo con el artículo 81.3.2 TFUE, en especial para el Derecho de familia. En otros supuestos el Consejo Europeo puede adoptar una decisión de ese tipo, también sin que los Parlamentos nacionales tengan un derecho de veto ${ }^{57}$.

54 Por ejemplo, Conseil constitutionnel 2007-560 DC, de 20 de diciembre de 2007. En la doctrina, respecto al principio democrático, también HUBER, VVDStRL 60 (2001), 194 (206 y siguiente).

55 LÜBBE-WOLF, VVDStRL 60 (2001), 246 (257 y siguiente).

$56 \mathrm{Al}$ respecto STCFA de 30 de junio de 2009 (nota 1), marginal 210.

57 Artículo 31.3 TUE, en materia de política exterior y de seguridad; artículo 312.2.2 TFUE, respecto al marco financiero de la UE; artículo 333.1 TFUE, respecto a la cooperación reforzada. Además, respecto a procedimientos legislativos que precisan la unanimidad con particularidades 
Al llevar a cabo la valoración constitucional, el TCFA diferencia entre cláusulas con un supuesto de hecho delimitado y cláusulas no delimitadas. En el segundo caso se exige una ley; en el primero la adopción de una decisión de los órganos legislativos ${ }^{58}$. Lo último sorprende porque este instrumento no está previsto en el artículo 23 GG. Por lo demás, el artículo 23.1.3 exige precisamente para estos casos, como compensación por la apertura material, una mayoría especial en el momento de la ratificación, como estableció el TCFA en la sentencia "Maastricht". Además, las "cláusulas-pasarela" en materia de Derecho de familia son tratadas como materialmente no delimitadas al llevar a cabo la subsunción, pero sin fundamentarlo, mientras que esto no ocurre en el caso de las disposiciones aplicables a la cooperación reforzada, repartidas por casi todos los capítulos del Tratado ${ }^{59}$. De este modo, los resultados del TCFA se corresponden "por casualidad" con la situación en Derecho europeo porque éste prevé un derecho de veto del Parlamento nacional en el caso de las cláusulas que el TCFA califica como insuficientemente determinadas, mientras que en el resto de los casos no. Ahora bien, los parámetros anteriormente definidos para los derechos nacionales de participación no son mantenidos de forma consecuente.

Un problema adicional se plantea en relación a las "cláusulas-pasarela" que incluyen un derecho de veto de los Parlamentos nacionales (artículo 48.7 TUE, artículo 81.3.2 TFUE). En este punto hay que darle la razón al TCFA al calificar las leyes de acompañamiento como inconstitucionales. De acuerdo con ellas, en el ámbito de aplicación de la legislación concurrente, el Bundestag y el Bundesrat podían, en principio, hacer uso del derecho de veto sólo si el otro daba su aprobación en virtud de normas que reproducían las exigencias de una resolución legislativa. Resulta convincente que eso no se sostiene desde el punto de vista del Derecho constitucional, que basta una decisión negativa de un órgano para que se produzca el veto ${ }^{60}$.

En principio, sin embargo, un derecho de veto es insuficiente, en opinión del TCFA. Del artículo 23.1.2 GG deriva la necesidad de una autorización legal al representante alemán en el Consejo Europeo para que éste emita su voto respecto a una correspondiente resolución del Consejo ${ }^{61}$. En este punto se hacen patentes diferentes concepciones de la democracia dentro de la UE: según la concepción (no sólo) británica lo decisivo es el "responsive government", no el "representative government “2 . Trasladado al presente caso: la (potencial) intervención de control, es decir, el derecho de veto, resultaría suficiente, sin

adicionales: artículo 153.2.4 TFUE, en materia de seguridad social; artículo 192.2 TFUE, respecto a determinadas medidas de política medioambiental; así como artículo 333.2 TFUE, respecto a la cooperación reforzada.

58 STCFA de 30 de junio de 2009 (nota 1), marginal 320.

59 STCFA de 30 de junio de 2009 (nota 1), marginal 319 por un lado, marginal 321 por otro.

60 STCFA de 30 de junio de 2009 (nota 1), marginal 415.

61 STCFA de 30 de junio de 2009 (nota 1), marginales 319 ss

62 Véase OLIVER, en: KLUTH (ed.), Europäische Integration und nationales Verfassungsrecht, 2007, pág. 163 (166). 
que se exija una legitimación positiva. La percepción alemana es en este punto más estricta ${ }^{63}$. En cualquier caso, si en el Tratado se prevé, por una parte, una resolución del Consejo Europeo y, por otra, se reconoce a los Parlamentos nacionales un derecho de veto, no se puede, sin vulnerar el Tratado, vincular el sentido del voto del representante en el Consejo a una autorización legal expresa del Parlamento nacional. Si bien los tratados de la Unión no contienen (salvo diversas garantías de independencia) ninguna norma relativa a la retroconexión nacional de los representantes de los Estados miembros, lo cierto es que de hecho se vulneran a pesar de todo las disposiciones de los Tratados $^{64}$. Si se exige una ley, el derecho de veto ya no tiene sentido. Formular a la vez exigencias a ese derecho de veto, como hace el TCFA, resulta contradictorio.

Quizá se exigió la ley sólo para asegurar la posibilidad de control por parte del Tribunal Constitucional respecto a si el «nivel de legitimidad es lo suficientemente elevado como para aceptar la decisión por mayoría " ${ }^{65}$. Si bien en este punto una ley parlamentaria no es en sí misma imprescindible, porque como alternativa se podría impugnar el voto favorable del representante del Gobierno, de hecho, sin embargo, éste resulta más difícilmente impugnable que una ley: antes de la adopción de la resolución no resulta apenas tangible, mientras que un veredicto negativo a posteriori por parte del Tribunal Constitucional no produciría ninguna consecuencia a nivel comunitario.

c) Sobre las cláusulas de "freno de emergencia"

Finalmente, en el ámbito del Derecho penal, un Estado miembro puede al menos evitar la adopción de una resolución vinculante para él cuando ésta afecte a aspectos fundamentales de su sistema de justicia penal (pese a la aplicación, en principio, del procedimiento legislativo ordinario) (artículos 82.3 y 83.3 TFUE, cláusulas de "freno de emergencia"). En ese caso sólo será posible una cooperación reforzada. Lo mismo se aplica a la coordinación de las prestaciones sociales en el contexto de la libre circulación de personas (subapartado segundo del artículo 48 TFUE). En este sentido, el TCFA exige que el Gobierno Federal haga uso de sus derechos «sólo de acuerdo con las instrucciones del Bundestag alemán" (y, en su caso, del Bundesrat) ${ }^{66}$. Esto resulta equívoco y sólo se comprende echando un vistazo a la resolución del Bundestag de acompañamiento a la ley del Tratado. Lo que se quiere decir es cla-

63 BVerfGE 83, 60 (78); de otra opinión TSCHENTSCHER, Legitimation der Dritten Gewalt, 2004, págs. 189 ss.

$64 \mathrm{Al}$ respecto recientemente (aunque en otro contexto) UERPMANN-WITTZACK/EDENHARTER, EuR 2009, 313 ss.

$65 \mathrm{Al}$ respecto STCFA de 30 de junio de 2009 (nota 1), marginal 321 (sobre el control por parte del Tribunal Constitucional también el marginal 243).

66 STCFA de 30 de junio de 2009 (nota 1), marginales 365, 400, 418. 
ramente que ante una resolución correspondiente del Bundestag, el Gobierno Federal ha de solicitar la suspensión del procedimiento legislativo ordinario comunitario; no se quiere decir que el Gobierno Federal no pueda hacer esto (y, por tanto, que no pueda evitar la adopción de una decisión a nivel europeo) sin resolución del Bundestag, como sugieren las palabras del TCFA. Además, de acuerdo con esto, el Gobierno Federal, cuando otro Estado utilice este instrumento y se discuta a nivel europeo una cooperación reforzada, podrá participar en la misma sólo con la aprobación del Bundestag. En el fondo esto resulta absolutamente convincente dado que a nivel europeo se trata, funcionalmente, de legislación.

\section{Sobre la participación del Bundestag en el Resto de los Casos}

Con la última observación se puede pasar al siguiente punto: a diferencia de lo que ocurrió en la sentencia sobre el Tratado de Maastricht ${ }^{67}$, el TCFA no aborda prácticamente la cuestión del papel de los órganos legislativos una vez que ya se ha efectuado la transferencia de derechos de soberanía, esto es, en particular cuando las instituciones comunitarias hacen uso de las competencias regulares. En aquella sentencia se mencionó al respecto el control parlamentario como fundamento de legitimidad. Frente a ello, el Tribunal Constitucional polaco ha subrayado, por ejemplo, que los derechos de participación correspondientes no suponen una expresión del control parlamentario del Gobierno, asignado exclusivamente al Sejm, la primera Cámara del Parlamento polaco, sino que serían un sustituto funcional de las competencias legislativas (atribuidas conjuntamente al Sejm y al Senado) que se pierden a nivel nacional ${ }^{68}$. Ahora al menos se hallan en los apartados 2 a 7 del artículo 23 GG las correspondientes normas. Las aspiraciones que vayan más allá de ello, como un derecho básico del Bundestag a impartir instrucciones al Gobierno Federal, tendrían como problemática consecuencia a la hora de adoptar decisiones por mayoría en Europa que se obstaculizaría en su caso que se pudiesen hacer valer los intereses alemanes porque no se implicaría al representante alemán en el Consejo en la búsqueda de una mayoría europea, al no tener aquél capacidad de negociación. En todo caso uno se podría haber imaginado que el TCFA estaría en desacuerdo al menos con el papel sobredimensionado del Bundesrat en comparación con el del Bundestag. Udo di Fabio, el Magistrado ponente del presente caso, había llamado la atención sobre este problema ya en $1993^{69}$, pero a día de hoy todavía no se ha resuelto. Por lo menos el legislador aprovecha

67 BVerfGE 89, 155 (191).

68 Sentencia de 12 de enero de 2005 (K 24/04); al respecto en detalle Baach, Parlamentarische Mitwirkung in Angelegenheiten der Europäischen Union, 2008, págs. 82 ss., 241.

69 DI FABIO, Der Staat 32 (1993), 191 (215); en el mismo sentido y en la misma época CLASSEN, ZRP 1993, 57 (60). 
la sentencia como ocasión para reforzar la posición del Bundestag respecto al Gobierno Federal también en relación a la actividad normativa cotidiana a nivel comunitario.

\section{EL ESTADO CONSTITUCIONAL DEMOCRÁTICO EN LA UNIÓN}

\section{Sobre el núcleo del Derecho constitucional Resistente A LA INTEGRACIÓN}

No es nuevo que el TCFA no acepta (ilimitadamente) la posición del TJCE acerca de los fundamentos de la aplicación nacional del Derecho europeo, especialmente acerca de la primacía de éste ${ }^{70}$. El TCFA se remite ahora también a la obligación de respeto al Derecho constitucional nacional, consolidada en el Tratado de Lisboa (actual artículo 4 TUE), y al asunto Kadi, resuelto por el $\mathrm{TJCE}^{71}$. Esto último no convence de todos modos. El Derecho de las Naciones Unidas no demanda ningún efecto directo en el ámbito jurídico de los Estados miembros, pero es que a las Naciones Unidas no se les han transferido derechos de soberanía. Aquí subyace la diferencia con el Derecho comunitario y de la Unión, cuyo efecto directo respecto a los ordenamientos ha hallado incluso anclaje explícito en el Tratado (actual artículo 249.2 TCE = artículo 288.2 TFUE). El TJCE ha deducido de esto (casi obligatoriamente) que del Derecho originario, de rango superior, no puede entonces afirmarse otra $\operatorname{cosa}^{72}$. Y como la transferencia de derechos de soberanía ha de ser "en principio revocable ${ }^{73}$, la participación de Alemania en la integración europea ha sido hasta ahora evidentemente inconstitucional, porque sólo con el Tratado de Lisboa se establece el derecho de retirada libre (artículo 50 TUE, en su nueva versión).

\section{SOBRE LA PRESERVACIÓN DE LA DEMOCRACIA NACIONAL}

\section{a) Fundamentos}

Que también el principio democrático pertenece al núcleo del Derecho constitucional resistente a la integración y que aquél a su vez exige que se

70 STCFA de 30 de junio de 2009 (nota 1), marginales 240 ss., 331 ss.; con anterioridad ya en las BVerfGE 73, 339 (387); 89, 155 (174 y siguiente); 102, 147 (163 y siguiente); sobre la crítica, CLASSEN, en: V. MANGOLDT/KLEIN/STARCK, Grundgesetz, vol. II, 5. ed., 2005, Art. 24, marginales 36 y siguiente.

71 STCFA de 30 de junio de 2009 (nota 1), marginales 339 y siguiente, en referencia también a la STJCE de 3 de septiembre de 2008 - asunto C-402/05 (Kadi); al respecto SCHMALENBACH, JZ 2009, 35.

72 STJCE, asunto 26/62, Rec. 1963, 1 (24 y siguiente) (van Gend \& Loos), ignorada por WIELAND, Neue Juristische Wochenschrift (NJW) 2009, 1841 (1842).

73 STCFA de 30 de junio de 2009 (nota 1), marginal 233. 
conserven a nivel nacional suficientes competencias decisorias es algo que podía deducirse ya de la sentencia sobre el Tratado de Maastricht ${ }^{74}$ y resulta como tal indiscutiblemente correcto. Nueva es la consecuencia que se extrae de ello de que determinadas decisiones políticas resultan especialmente delicadas también desde un punto de vista democrático por su relevancia para los derechos de los ciudadanos, y la deducción que de ahí se extrae de que en cinco ámbitos concretos una integración debería ser posible sólo dentro de unos márgenes muy estrictos, dada la limitada legitimidad democrática de la $\mathrm{UE}^{75}$

En este punto confunde ante todo que, como se aprecia con frecuencia en este sentido, no se trata de elementos específicos de la estatalidad constitucional alemana como, por ejemplo, el federalismo o la notable significación de los derechos fundamentales en el ordenamiento jurídico, y como se conocen también en otros Estados miembros, por ejemplo Francia, con la tradición del laicismo y del "service public", sino que se trata de un problema que se da en todos los Estados miembros de la UE. Pese a todo, la posición del TCFA no tiene en este punto parangón en Derecho comparado ${ }^{76}$. Erróneo resulta sobre todo el nivel de detalle de las reflexiones; al final de lo que se trata en definitiva es del artículo 79.3 GG. El Tribunal constitucional checo, que recientemente, después del TCFA, ha tenido que decidir por segunda vez sobre el Tratado de Lisboa y que en el pasado había citado la jurisprudencia del TCFA relativa a los límites constitucionales a la integración europea, compartiendo su postura ${ }^{77}$, ha rechazado de manera consecuente, apelando a los límites funcionales de la jurisdicción, defender un canon determinado similar de ámbitos resistentes a la integración ${ }^{78}$. La justificación política de la exigencia de moderación en los ámbitos correspondientes no legitima restricciones, resistentes a la reforma constitucional, de la libertad de los otros órganos constitucionales para conformar la realidad. El TCFA hace que se eche aquí de menos toda orientación prospectiva. Quizá lo único que se perseguía con esta exigencia era hacer referencia a la posibilidad de plantear (a través del artículo 146 GG) consultas populares en cuestiones de integración europea. Pero, con independencia de cómo se valore esto desde el punto de vista de la política jurídica, habría que haber dejado su introducción en manos del poder legislativo (normal) de reforma de la Constitución, porque la Ley Fundamental no conoce ninguna "reforma total" según el modelo austríaco (artículo 44 de la Constitución Federal de ese país). La europeizacion del Derecho pe-

74 STCFA de 30 de junio de 2009 (nota 1), marginales 249 ss., en referencia a BVerfGE 89, 155 (207 ss.).

75 STCFA de 30 de junio de 2009 (nota 1), marginales 249 ss. y 351 ss.

76 Críticos por eso también OPPERMANN, Europäische Zeitschrift für Wirtschaftsrecht (EuZW) 2009, 473; CHR. CALLIES Y NETTESHEIM, los dos en el Frankfurter Allgemeine Zeitung de 27 de agosto de 2009, pág. 8.

77 Sentencia de 26 de noviembre de 2008, PL Ús 19/08, marginales 116 ss.

78 Sentencia de 3 de noviembre de 2009, PL ÚS 29/09. 
nal (supuestamente "resistente en principio a la integración europea" ${ }^{79}$ ), por ejemplo, no constituye el supuesto contemplado en el artículo 146 GG. Finalmente, estas reflexiones no eran en modo alguno necesarias. Una referencia a los correspondientes ámbitos particularmente delicados, mencionando los respectivos límites que señala el Tratado, habría bastado para constatar que el Tratado no plantea en este sentido ningún problema.

\section{b) Los ámbitos concretos}

Dos de los cinco ámbitos pertenecen (también) al campo del Derecho de la organización del poder público; en este sentido, las reflexiones como tales resultan en cierta medida plausibles. El primero concierne a las decisiones respecto a la intervención de la fuerza militar ${ }^{80}$. La afirmación en la parte de la sentencia dedicada a los razonamientos teóricos y abstractos se refiere expresamente en cualquier caso sólo al Ejército alemán (Bundeswehr); qué valoración merecería un Ejército integrado como el que se contemplaba en los artículos 9 y siguientes del Tratado sobre la Comunidad Europea de Defensa de 26 y 27 de mayo de $1952^{81}$ es una cuestión que no se aborda expresamente. La parte de la sentencia en la que se lleva a cabo la subsunción permite sin embargo deducir que, presumiblemente, también se alude a un Ejército de ese tipo. Allí se habla concretamente de soldados alemanes; además se califica al artículo 24.2 GG de lex specialis respecto al artículo 23 GG $^{82}$. Esta última afirmación habría encajado mejor de todos modos en la parte de la sentencia dedicada a los razonamientos teóricos y abstractos. Dentro de la soberanía financiera entra, según el TCFA, el que el legislador nacional pueda decidir en última instancia acerca del total de cargas que asumen los ciudadanos $^{83}$. De este modo no se excluyen (probablemente) los impuestos de la Unión, sino sólo una competencia exclusiva (nada realista) de la Unión en este terreno.

Junto a ello menciona el TCFA la conformación de las relaciones vitales desde la perspectiva del Estado social (sozialstaatliche Gestaltung von Lebensverbältnissen ${ }^{84}$. Como la Ley Fundamental obliga a que también la integración europea respete el Estado social, a la política no le queda más remedio que hacer equilibrios. En concreto destaca el Tribunal la jurisprudencia del TJCE relativa a la dimensión social de la integración europea. Al tratar el tema de los límites de la ciudadanía europea en el ámbito de la cuestión referente a si todavía hay un pueblo estatal, se menciona también la jurisprudencia del

79 STCFA de 30 de junio de 2009 (nota 1), marginal 363.

80 STCFA de 30 de junio de 2009 (nota 1), marginales 254 y siguiente, 381 ss.

81 BGBl. 1952 II 343.

82 STCFA de 30 de junio de 2009 (nota 1), marginales 382 ó 388.

83 STCFA de 30 de junio de 2009 (nota 1), marginal 256.

84 STCFA de 30 de junio de 2009 (nota 1), marginales 257 ss., 392 ss. 
TJCE sobre los derechos sociales que se derivan de esta ciudadanía comunitaria, sobre todo la sentencia en el asunto Förster, relativa a la obligación de conceder ayudas al estudio también a los extranjeros ${ }^{85}$, que subraya más claramente que otras sentencias los límites de estos derechos.

En Derecho penal (incluido el Derecho procesal), «a este ámbito particularmente delicado en relación a los derechos fundamentales" se le debe permitir una armonización "sólo respecto a determinados asuntos transfronterizos y en condiciones restrictivas ${ }^{86}$. Esto sorprende. Al menos el Derecho procesal penal viene actualmente codeterminado en nada despreciable medida por el Convenio Europeo de Derechos Humanos, y en una comunidad de valores no puede tampoco un acuerdo sobre mínimos éticos representar un problema fundamental. Y mientras que el artículo 83.1 TFUE habla de ámbitos delictivos que, debido a circunstancias que se explican con más detalle, tengan una "dimensión" transfronteriza, el TCFA reduce la competencia a la "criminalidad transfronteriza ${ }^{87}$ (¿exigida en cada caso concreto?). Esto último haría en su caso necesario un Derecho penal fraccionado (uno para delitos que tengan una pura dimensión nacional, otro para delitos transfronterizos). Con ello se iría en contra de la exigencia de concreción de los supuestos de hecho exigida por el Estado de Derecho (¿cuándo se pueden prever los efectos transfronterizos de un delito?). Una exigencia (supuestamente) fundamentada en la democracia conduce de este modo a una inseguridad dudosa desde el punto de vista del Estado de Derecho. Ello no habría sido necesario para fundamentar el derecho de los órganos legislativos nacionales «a impartir instrucciones, ${ }^{88}$ que se defiende.

Finalmente se mencionan ámbitos relevantes desde el punto de vista cultural, como el sistema educativo, el Derecho de familia, el Derecho de la religión y, en parte, el Derecho de los medios de comunicación ${ }^{89}$. En la parte de la sentencia en la que se lleva a cabo la subsunción no se vuelve a aludir a ellos. Aparentemente no plantea el Tratado de Lisboa problemas en este sentido. Respecto al Derecho de familia incorpora éste la garantía procedimental de la unanimidad (artículo 67.5 TCE y ahora artículo 81.3 TFUE; nueva es solamente la "cláusula-pasarela" del artículo 81.3.2 TFUE), respecto al ámbito educativo la garantía material de la prohibición de armonización (artículos 149 y 150 TCE $=$ artículos 165 y 166 TFUE, apartados 1 y 4 en cada caso), reforzada ahora aún más por la prohibición de aplicación del artículo 352 TFUE (apartado 4). Asimismo contiene el Tratado de Lisboa garantías adicionales respecto a la religión (artículo 17 TFUE). Pero quizá de lo que se trataba en este punto era solamente de crear un instrumento para poder oponer

85 STCFA de 30 de junio de 2009 (nota 1), marginal 350, en referencia a la STJCE de 18 de noviembre de 2008, asunto C-158/07 (Förster).

86 Las citas en STCFA de 30 de junio de 2009 (nota 1), marginal 253 (similares 352 ss.) así como en el marginal 363.

87 STCFA de 30 de junio de 2009 (nota 1), marginal 352; cfr. también el marginal 253.

88 STCFA de 30 de junio de 2009 (nota 1), marginal 365; al respecto supra, III.3.c.

89 STCFA de 30 de junio de 2009 (nota 1), marginal 260 
constitucionalmente algo a determinados desarrollos que se están produciendo dentro del Derecho de la Unión. Respecto al Derecho de la religión hay que mencionar en especial el Derecho en materia de lucha contra la discriminación ${ }^{90}$, que, igual (palabra clave: “discriminación por motivo de orientación sexual", al respecto también artículo 21 de la Carta de Derechos Fundamentales ${ }^{91}$ ) que la ciudadanía de la Unión (palabra clave: «derecho al nombre, ${ }^{92}$ ), influye cada vez más en el Derecho de familia. En el Derecho de los medios de comunicación los intentos de la Comisión de imponer límites, más allá de las normas sobre las ayudas estatales, a la expansión hacia Internet de los servicios públicos de radiodifusión financiados por medio de tasas desempeñaron probablemente algún papel ${ }^{93}$.

Sin que se aluda a esto en mayor detalle en la parte de la sentencia dedicada a los razonamientos teóricos y abstractos, la política del comercio exterior se menciona finalmente al llevar a cabo la subsunción. Es destacable en este punto la obligación, que no se justifica más detalladamente pero que en definitiva convence, de informar al Parlamento sobre los desarrollos que se produzcan en el marco de la Organización Mundial de Comercio ${ }^{94}$. Esta declaración se construye de manera susceptible de ser ampliada y puede significar (con razón) que el Gobierno Federal en el futuro habrá de informar a los órganos legislativos acerca de todos los desarrollos en el ámbito de las organizaciones internacionales que afecten a sus competencias. Una extensión de los derechos del Bundestag a otros ámbitos puede suponer una razonable contribución a la disminución de la pérdida de legitimidad que se asocia a la creciente densificación de las relaciones internacionales ${ }^{95}$.

\section{Sobre el papel del Tribunal Constitucional}

En definitiva, el TCFA reclama para sí una amplia competencia de control frente a la ulterior integración ${ }^{96}$. El que no se deje sólo en manos del TJCE el

90 El artículo 4.2 de la Directiva 2000/78 permite un trato diferenciado en atención a la pertenencia a una comunidad religiosa sólo, como muestra una comparación sistemática de los dos subapartados de la norma, si está relacionada con la actividad que se ha de desempeñar, y está de esta manera en contradicción con la comprensión clásica alemana (al respecto la BVerfGE 70, 138 [164 ss.]).

$91 \mathrm{Al}$ respecto, por ejemplo, STJCE, asunto C-267/06, Rec. 2008, I-1757 (Maruko) = JZ 2008, 787, con comentario de BRINKTRINE, y opuesta en su contenido a la sentencia (de sección) del TCFA, JZ 2009, 792, con un comentario también crítico en este extremo de CLASSEN. La decisión del TCFA de 7 de julio de 2009 (número de acta: 1 BvR 1264/07) le da, sin embargo, la razón ahora al TJCE.

92 STJCE, asunto C-148/02, Rec. 2003, I-11613 (García Avello); STJCE de 14 de octubre de 2008, asunto C-353/06 (Grunkin-Paul) = JZ 2009, 151, con comentario de KROLL-LUDWIGS.

$93 \mathrm{Al}$ respecto OPPERMANN/CLASSEN/NETTESHEIM (nota 44), marginales 26/37.

94 STCFA de 30 de junio de 2009 (nota 1), marginal 375.

95 STCFA de 30 de junio de 2009 (nota 1), marginal 376; en este sentido también POSCHER, VVDStRL 67 (2008), 160 (186 ss.); CLASSEN, ibidem, pág. 365 (384).

96 Véase sobre todo la STCFA de 30 de junio de 2009 (nota 1), marginales 240 ss., 336 ss. 
ver si se respetan los límites competenciales en este contexto se corresponde con la jurisprudencia reiterada tanto del TCFA como de otros Tribunales nacionales superiores ${ }^{97}$. Sin embargo, uno se habría alegrado si el TCFA además de la noción de soberanía, no mencionada en el texto de la Ley Fundamental, hubiese aludido al compromiso con la jurisdicción internacional contenido expresamente en su artículo 24.3 (algo poco habitual, en la UE sólo aparece también en las Constituciones de Irlanda y Portugal), cuyo sentido es precisamente reconocer el derecho a que sea una instancia internacional quien decida en último lugar. En vez de eso se mencionan sólo los apartados 1 y 2 del artículo 24 al describir la apertura internacional de la Ley Fundamental ${ }^{98}$, dejando el apartado 3 conscientemente de lado. Al menos terminológicamente aparece, en lugar de la dramática expresión "acto jurídico que transgrede los límites competenciales" (ausbrechender Rechtsakt) (mencionada sólo al citar literalmente la sentencia "Maastricht"), la noción, conocida del Derecho internacional público, del acto ultra vires ${ }^{99}$. Es importante también la asunción de este control por el TCFA en régimen de monopolio, de forma análoga a lo dispuesto en el artículo 100.1 GG, para asegurar la capacidad funcional del "ordenamiento jurídico comunitario" ${ }^{100}$

Sorprendente y superflua resulta, por el contrario, la referencia a que el legislador podría prever en este punto un procedimiento especial. Tal cosa sería contraria al Derecho europeo porque la magnitud de las competencias transferidas está regulada en los Tratados de integración y el TJCE ha sido llamado en esa medida, con el consentimiento de los órganos legislativos alemanes, a decidir en última instancia; también en ese sentido se han transferido derechos de soberanía ${ }^{101}$. Si el TCFA calificase una sola vez un acto soberano europeo como acto ultra vires, eso sería quizá, en el caso concreto, todavía soportable; pero si, por el contrario, el legislador alemán regulase en abstracto, sin necesidad, un supuesto de ese tipo, provocaría un conflicto con la UE no sólo apenas susceptible de solución sino, sobre todo, también bastante innecesario.

Esto preocupa ante todo porque el TCFA interpreta los Tratados de la Unión en numerosos puntos de una forma que cabría calificar por lo menos

97 BVerfGE 58, 1 (29); 59, 63 (85); 75, 223 (242); 89, 155 (188). Sobre el extranjero véanse, aparte de la sentencia del Tribunal Constitucional checo citada en la STCFA de 30 de junio de 2009 (nota 1), marginal 338, por ejemplo la sentencia del Tribunal Supremo danés (EuGRZ 1999, 49) y la del Tribunal Constitucional polaco (EuR 2006, 236 [243]). Véanse además, acerca de Suecia y Hungría, los trabajos de NERGELIUS $(\$ 22$, marginal 21 ) y SONNEVEND ( $\$ 25$, marginales 24 y siguiente), ambos en: V. BOGDANDY/CRUZ VILLALÓN/HUBER (eds.), Handbuch Ius Publicum Europaeum, vol. II, 2009. Respecto a la crítica constitucional CLASSEN, en: V. MANGOLDT/KLEIN/STARCK (nota 70), Art. 24, marginal 52; además Lenz, Frankfurter Allgemeine Zeitung de 8 de agosto de 2009, pág. 7; PACHE EuGRZ 2009, 285 (297 y siguiente).

98 STCFA de 30 de junio de 2009 (nota 1), marginal 222.

99 STCFA de 30 de junio de 2009 (nota 1), marginal 240. Al respecto en la doctrina por ejemplo KLEIN, en: GRAF VITZTHUM (ed.), Völkerrecht, 4. ed., 2007, marginal IV 98, 192 ss.

100 STCFA de 30 de junio de 2009 (nota 1), marginal 241. Se refiere seguramente al ordenamiento jurídico de la Unión.

101 Ignorado en lo fundamental por P. KIRCHHOF, JZ 1998, pág. 965. 
de discutible. Ciertamente la interpretación de éstos es necesaria para decidir acerca de su constitucionalidad. Ahora bien, mientras que en determinados casos se deja claro que no se aspira en ese punto a una decisión vinculante ${ }^{102}$, una interpretación más que dudosa del Derecho de la Unión por parte del TCFA constituye en otros casos el fundamento de la decisión. Esto afecta, por ejemplo, al papel de los Parlamenos nacionales de acuerdo con el artículo 48.7 TUE (supra, III.3.c), el sujeto de representación del Parlamento Europeo, incluidos los defectos del Derecho electoral (supra, II.1.c) o las competencias de la UE en el ámbito del Derecho penal (supra, IV.2.b). El TCFA recurre aquí al instrumento de la interpretación conforme a la Constitución, explícitamente en el contexto de las competencias sobre el Derecho penal ${ }^{103}$; por lo demás, el Tratado satisface las exigencias constitucionales (ihabría que añadir aquí "sólo"?) "conforme a los fundamentos que sustentan el fallo" (nach Maßgabe der Gründe $)^{104}$. A falta de eficacia vinculante de las sentencias del TCFA para el TJCE puede ser que esto no convenza. Incluso el recurso de emergencia, concretamente declarar los actos jurídicos de la Unión en tanto que (presuntos) actos ultra vires como no aplicables internamente, puede operar sólo en parte, específicamente respecto a actos jurídicos concretos. Si se trata de cuestiones institucionales y de las concepciones fundamentales que a ello se asocian, el TCFA carece de poder. Y una reserva, discutida en parte, referida a la sentencia del TCFA en el marco de la ratificación sería inadmisible porque es incompatible con el derecho del TJCE a decidir en último lugar previsto en el Tratado, es decir, con la esencia del Tratado, de modo que una declaración en ese sentido carecería, por tanto, de eficacia. Al menos el TCFA no remite en el texto a los fundamentos del fallo, a diferencia de lo que hizo en la sentencia relativa al Tratado internacional sobre los fundamentos de las relaciones entre la República Federal y la República Democrática alemanas (Grundvertragsurteil) $^{105}$.

\section{VALORACIÓN GLOBAL}

Si uno se pregunta qué impulsos reciben el Derecho europeo y el Derecho constitucional europeo de la nueva sentencia del TCFA, la primera mirada recae sobre el fortalecimiento, que se vincula a la sentencia, del papel del Bundestag. Este fortalecimiento es en principio importante y correcto. También las consecuencias concretamente formuladas (por ejemplo respecto al artículo 352 TFUE) son en esta medida al menos plausibles, si bien en algunos ámbitos uno podría haber esperado todavía más. Frente a ello el TCFA, con su

102 STCFA de 30 de junio de 2009 (nota 1), marginal 305 sobre el recurso de subsidiariedad, marginal 379 sobre la competencia de la UE en materia de inversiones extranjeras directas.

103 STCFA de 30 de junio de 2009 (nota 1), marginal 362.

104 STCFA de 30 de junio de 2009 (nota 1), marginal 273, en sentido similar marginal 420.

105 BVerfGE 36, 1 (3). 
concepción amplia del artículo 23.1.2 GG, provoca simplemente caos en la dogmática (también del artículo 24.1 GG). Al final resulta que el Bundesrat (lo que es contraproductivo desde el punto de vista de los recurrentes) resulta fortalecido en perjuicio del Bundestag. Esto convence tan poco como los límites de amplio alcance a la integración. La impresión que transmite la sentencia de que el Tratado de Lisboa, ratificado entretanto por casi todos los demás Estados miembros de la UE, es constitucional por poco, no satisface la obligación de la República Federal Alemana de contribuir a la Unión Europea según el artículo 23 GG. Sorprendentes resultan, finalmente, las numerosas lecciones en materia de concepción democrática que imparte el Tribunal Constitucional alemán (también a otros Estados miembros de la UE).

En conjunto intenta el TCFA sobre todo, frente al tinte democrático de la sentencia, capturar la dinámica de la integración con los medios del Derecho constitucional alemán, y no tanto, por el contrario, salvaguardar su legitimación político-democrática en el día a día. A la jurisprudencia del Tribunal de Luxemburgo, favorable a la integración y sin duda no siempre muy fiel al tenor literal, ha opuesto el TCFA por lo demás una sentencia todavía menos fiel al tenor literal, todavía más referida a la soberanía que las sentencias de otros Tribunales constitucionales relativas al Tratado de Lisboa y sustentada además en parámetros que no pueden eludirse mediante simples reformas constitucionales. Incluso aunque la soberanía no se entienda ahí como derecho exclusivo sino "Sólo" como derecho de amplio alcance para decidir en última instancia, esto no resulta convincente. Después de todo Alemania pudo recuperar su soberanía tras la guerra y la caída del Muro sólo gracias a su integración en la Comunidad Europea ${ }^{106}$. El Tribunal no hace justicia a esta dimensión histórica de la integración europea.

TITLE: ¿Bundestag's legitimate strengthening or Procusto's constitutional bed? About the Lisbon Treaty's Constitutional Federal Court judgment.

ABSTRACT: The Constitutional Court Decision about the Lisbon Treaty claims a more important role for national parliament in the European integration process. Nevertheless, the Court thinks more in terms of transfer of powers than in the exercise of them. The Court Decision follows the interpretation given in his previous decision about the Maastricht Treaty ("Maastricht Decision"), although introduces some precisions. From its reading one might think that the Court is mainly interesting in dress the dynamic of the European integration with legal instruments.

RESUMEN: La sentencia del Tribunal Constitucional Federal alemán sobre el Tratado de Lisboa (Juristenzeitung 2009, 890) reivindica con razón un papel más importante de los Parlamentos nacionales en el proceso de integración europea. Abora bien, el Tribunal Constitucional Federal tie-

106 El artículo 7.2 del Tratado de 26 de mayo de 1952 que regulaba las relaciones de la República Federal Alemana con las tres potencias aliadas (Deutschlandvertrag), en la versión de 23 de octubre de 1954, hablaba incluso expresamente de una Alemania reunificada que "esté integrada en la Comunidad Europea" (BGBl. 1955 II 306). Crítico por eso también LENZ, Frankfurter Allgemeine Zeitung de 8 de agosto de 2009, pág. 7. 
ne en mente en este sentido sobre todo la transferencia de competencias, apenas el ejercicio de las mismas. Por lo demás, la sentencia se mueve en muchos puntos en la línea de la sentencia "Maastricht, aunque aporta en todo caso numerosas precisiones (en parte plenas de sentido; en numerosas ocasiones, sin embargo, cuestionables) y ocasionalmente cautelosos desarrollos ulteriores. Al final a uno le da la impresión de que el Tribunal Constitucional Federal intenta sobre todo, frente al tinte democrático que impregna la sentencia, capturar la dinámica de la integración europea con instrumentos juridicos, y no tanto salvaguardarla de verdad desde el punto de vista político-democrático.

Key wORDS: Republic of Germany. Fundamental Law of Bonn. Federal Constitutional Court. European Union. Democratic legitimacy. Treaty of Lisbon.

Palabras Clave: República Federal de Alemania., Ley Fundamental de Bonn. Tribunal Constitucional Federal. Unión Europea. Legitimidad democrática. Tratado de Lisboa. 